\title{
Cost-Effectiveness of Ribociclib plus Letrozole Versus Palbociclib plus Letrozole and Letrozole Monotherapy in the First-Line Treatment of Postmenopausal Women with HR+/HER2- Advanced or Metastatic Breast Cancer: A U.S. Payer Perspective
}

\author{
Rohit Mistry, MPharm, MBA, MSc; Jessica R. May, PhD; Gaurav Suri, MSc, BTech; \\ Kate Young, PhD, MPH; Diana Brixner, PhD, RPh, FAMCP; Gary Oderda, PharmD, MPH; \\ Joseph Biskupiak, PhD, MBA; Derek Tang, PhD, BSPharm; Subrata Bhattacharyya, MSc; \\ Dinesh Mishra, PhD, MSc; Devarshi Bhattacharyya, MPH, MSc, BDS; and Anand A. Dalal, PhD, MBA
}

\begin{abstract}
BACKGROUND: U.S. regulatory approvals of the cyclin-dependent kinase 4 and 6 (CDK 4/6) inhibitors ribociclib and palbociclib as add-ons to letrozole greatly enhance the prospects for treating postmenopausal women with hormone receptor-positive $(\mathrm{HR}+)$ /human epidermal receptor 2-negative (HER2-) advanced or metastatic breast cancer. Clinical trials have established that the combination of a CDK 4/6 inhibitor with letrozole can significantly improve progression-free survival (PFS) versus letrozole monotherapy and is safe and well tolerated. Cost-effectiveness studies are required to inform payers and clinical decision makers on the money value of combination treatment in clinical practice.
\end{abstract}

OBJECTIVE: To evaluate the cost-effectiveness of ribociclib plus letrozole versus palbociclib plus letrozole and versus letrozole monotherapy in the first-line treatment of postmenopausal women with HR+/HER2- advanced or metastatic breast cancer from a U.S. private third-party payer perspective.

METHODS: A partitioned survival model including 3 health states (progression free, with either overall response or stable disease; progressed disease; and death) simulated lifetime costs and outcomes over a 40-year lifetime horizon with a 1-month cycle length. Clinical efficacy data (PFS and overall survival [OS]) were derived from a phase III trial of ribociclib plus letrozole (MONALEESA-2; NCT01958021), a phase II trial of palbociclib plus letrozole (PALOMA-1; NCT00721409), and a Bayesian network metaanalysis. Health care costs included drug acquisition and monitoring, disease management, subsequent therapies, and serious drug-related adverse events. Effectiveness was measured in life-years, derived from survival projections, and in quality-adjusted life-years (QALYS), calculated from time spent in each state combined with health-state utility values. A one-way deterministic sensitivity analysis explored the impact of uncertainty in key model parameters on results, and probabilistic uncertainty was assessed through a Monte Carlo probabilistic sensitivity analysis.

RESULTS: Ribociclib plus letrozole was dominant versus palbociclib plus letrozole, with a cost saving of $\$ 43,037$ and a gain of 0.086 QALYs. Compared with letrozole monotherapy, ribociclib plus letrozole was associated with an incremental cost of $\$ 144,915$ and an incremental QALY of 0.689 , equating to an incremental cost-effectiveness ratio of $\$ 210,369$ per QALY. Key model drivers included OS HRs for palbociclib plus letrozole versus letrozole and for ribociclib plus letrozole versus letrozole, the PFS HR for palbociclib plus letrozole versus letrozole, PD health-state costs, utility of response, and cost discount rate. The probabilities that ribociclib plus letrozole was cost-effective versus letrozole at thresholds of $\$ 50,000$, $\$ 100,000$ and $\$ 200,000$ per QALY gained were $1.6 \%, 6.3 \%$, and $50.5 \%$, respectively.
CONCLUSIONS: In the United States, ribociclib plus letrozole is a costeffective alternative to palbociclib plus letrozole for the first-line treatment of postmenopausal women with HR+/HER2- advanced or metastatic breast cancer. Ribociclib plus letrozole is also cost-effective versus letrozole monotherapy at willingness-to-pay thresholds greater than $\$ 198,000$ per QALY (for probabilistic analysis).

J Manag Care Spec Pharm. 2018;24(6):514-23

Copyright $\odot 2018$, Academy of Managed Care Pharmacy. All rights reserved.

\section{What is already known about this subject}

Current treatment options for postmenopausal women with hormone receptor-positive (HR+)/human epidermal receptor 2-negative (HER2-) advanced breast cancer include aromatase inhibitors (e.g., letrozole), selective estrogen receptor modulators (e.g., tamoxifen), estrogen receptor antagonists (e.g., fulvestrant), and the cyclin-dependent kinase 4 and 6 (CDK 4/6) inhibitors (e.g., palbociclib and ribociclib).

The MONALEESA-2, PALOMA-1, and PALOMA-2 trials have demonstrated that adding the CDK $4 / 6$ inhibitors ribociclib or palbociclib to letrozole is a safe and efficacious alternative to letrozole monotherapy for first-line postmenopausal HR+/HER2advanced breast cancer.

\section{What this study adds}

This is the first study to assess the cost-effectiveness of ribociclib in combination with the aromatase inhibitor letrozole versus palbociclib plus letrozole and versus letrozole monotherapy from a U.S. private third-party payer perspective.

The results indicate that ribociclib plus letrozole is cost-effective compared with palbociclib plus letrozole as the first-line treatment of postmenopausal women with HR+/HER- advanced or metastatic breast cancer, resulting in gains in quality-adjusted life-years and cost savings. 


\section{Cost-Effectiveness of Ribociclib plus Letrozole Versus Palbociclib plus Letrozole and Letrozole Monotherapy in the First-Line Treatment of Postmenopausal Women with HR+/HER2- Advanced or Metastatic Breast Cancer: A U.S. Payer Perspective}

I $\mathrm{n}$ the United States, $72.7 \%$ of invasive breast cancer cases are observed in patients with hormone receptor-positive (HR+)/human epidermal receptor 2-negative (HER2-) breast cancer. ${ }^{1}$ For this population, the mainstay of treatment is hormonal therapy, which may include aromatase inhibitors (e.g., letrozole), selective estrogen receptor modulators (e.g., tamoxifen), and estrogen receptor antagonists (e.g., fulvestrant). ${ }^{2,3}$ However, clinical resistance to hormonal therapy due to increased expression of growth factor receptor pathways eventually occurs in most patients, leading to disease progression. ${ }^{4}$

Cyclin-dependent kinase (CDK) 4 and 6 inhibitors (e.g., palbociclib, ribociclib) work by hindering phosphorylation of the retinoblastoma protein, a key regulator of cell cycle progression, thereby inhibiting tumor growth. ${ }^{5}$ Several clinical studies have shown that the combination of ribociclib or palbociclib with letrozole is a tolerable, safe, and efficacious alternative to letrozole monotherapy for first-line HR+/HER2advanced breast cancer. ${ }^{6-8}$ In the MONALEESA-2 study, for example, ribociclib plus letrozole prolonged progression-free survival (PFS) by 7.1 months, reducing the rate of progression or death by $44 \%$ compared with letrozole plus placebo (hazard ratio $[\mathrm{HR}]=0.56 ; 95 \%$ confidence interval $[\mathrm{CI}]=0.43-0.72$; $P<0.0001){ }^{6}$

Along with providing health benefits, the use of ribociclib/ palbociclib plus letrozole versus letrozole monotherapy may increase drug expenditure and health care costs due to longer PFS and life expectancy. The extent to which these additional costs are considered a judicious use of resources is evaluated by comparing the costs and effectiveness of competing treatment strategies and summarizing the incremental cost per unit of health gained. Cost-effective analyses are central to the reimbursement process in countries such as the United Kingdom (UK), Sweden, and Canada but historically have had limited use in the United States. Through the work of the Panel on Cost-effectiveness in Health and Medicine and independent organizations such as the Institute for Clinical and Economic Review, cost-effectiveness analyses are taking a more central role in U.S. treatment coverage decisions.

The incremental cost-effectiveness of palbociclib plus letrozole versus letrozole monotherapy has been reported elsewhere, with results suggesting that palbociclib plus letrozole is not cost-effective versus letrozole monotherapy in postmenopausal HR+/HER2- advanced breast cancer. ${ }^{9-11}$ To inform decision makers regarding the cost-effectiveness of ribociclib compared with palbociclib in the same setting, a cost-effectiveness analysis of ribociclib plus letrozole versus palbociclib plus letrozole and versus letrozole monotherapy was conducted.

\section{Methods}

\section{Model Overview}

A partitioned survival model was developed in Microsoft Excel 2010 to estimate costs, life-years (LYs), and quality-adjusted
LYs (QALYS) associated with treatment with ribociclib plus letrozole, palbociclib plus letrozole, and letrozole monotherapy from a U.S. third-party payer perspective representing both commercial and Medicare lives. The analysis was performed on the intention-to-treat cohort of MONALEESA-2, comprising postmenopausal women with HR+/HER2- advanced or metastatic breast cancer. ${ }^{6}$ This covers the full indication for ribociclib in the United States. ${ }^{12}$

Consistent with approaches used in other advanced oncology indications, the model uses survival curves for PFS and overall survival (OS) to directly estimate state occupancy over time, without the need for transition probabilities between health states as required for Markov models. ${ }^{13}$ The area under the OS curve estimates the proportion of patients who are alive, and the area under the PFS curve estimates the proportion of patients who are alive and progression free (PF). The difference between OS and PFS gives the number of patients who are alive and with progressed disease (PD).

The model comprised 3 health states: PF (with either overall response or stable disease, defined by investigator assessment), $\mathrm{PD}$, and death (Figure 1). The substates of response and stable disease captured the quality-of-life (QoL) benefits of tumor reduction, while the PD state captured the cost and QoL implications of tumor progression, including worsening of symptoms and the use of subsequent treatment lines.

All patients entered the model in the PF with stable disease state and immediately commenced treatment. From this state, patients could achieve a complete or partial response to therapy and enter PF with response, experience progression and enter PD, or die and enter death. From PD, patients could enter the death state only. All deaths were modeled, including those unrelated to the disease.

Patient transitions and length of stay among the PF, PD, and death states were used to calculate the expected costs and effectiveness of treatment over a 40-year lifetime horizon. A cycle length of 1 month was used in line with the dosing schedule for ribociclib and palbociclib, which comprises 21 days on drug followed by 7 days off drug. ${ }^{12,14}$ Half-cycle (mid-cycle) corrections were applied to both costs and effectiveness as per standard guidelines. The model incorporated subsequent (second-and third-line) therapies for patients who progressed while on treatment; the subsequent use of a CDK 4/6 inhibitor was considered clinically inappropriate.

\section{Treatment Efficacy and Duration}

For letrozole monotherapy, PFS and OS were modeled from the control arms of MONALEESA-2 (PFS and overall response) and PALOMA-1 (OS). 6,7 For PFS, Kaplan-Meier probabilities were used for the initial 22 months (end of follow-up), with lifetime extrapolations based on parametric models fitted to patient-level data. The preferred parametric model for the base case was Weibull, which demonstrated the best fit to survival 
FIGURE 1 Health-State Structure of the Economic Model $^{\mathrm{a}}$

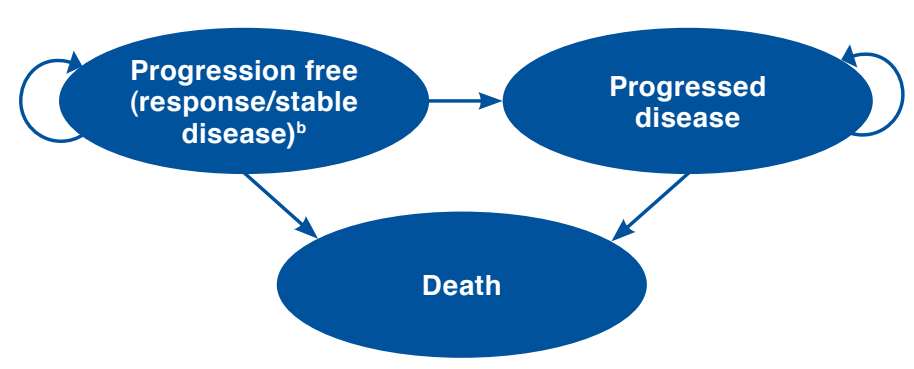

a Health-state transitions are not explicitly modeled in the partitioned survival analysis. The direction of transition in the model is provided as an illustration. bProgression free is split into 2 substates and the patient's transition between complete response/partial response and stable disease.

within MONALEESA-2 based on Akaike Information Criteria and prediction of the Kaplan-Meier plot and generated a plausible prediction of 10 -year PFS (0.01\%), according to independent clinical expert validation.

OS from MONALEESA-2 was relatively immature at the time of model development (20 events/334 persons). When modeled using Kaplan-Meier probabilities (up to 22 months) followed by parametric models, MONALEESA-2 generated 10 -year survival rates for letrozole that ranged from 19\% to $72 \%$. These predictions greatly exceeded real-world estimates of survival at the time (6.4\% to $12 \%$ ) and lacked plausibility, according to independent clinical review. ${ }^{15,16}$ To improve model predictions, the OS Kaplan-Meier from the letrozole arm of PALOMA-1 was used in place of data from MONALEESA-2 (up to 33 months), with lifetime extrapolations based on parametric models fitted to OS from the letrozole arm of MONALEESA-2. As with PFS, a Weibull distribution demonstrated the best fit to OS and yielded a plausible 10-year survival rate of $11 \%$, when combined with the PALOMA-1 data.

For ribociclib plus letrozole, PFS was estimated from the active arm of MONALEESA-2 (PFS and overall response) and OS from the active arm of PALOMA-1 (OS), using the same methods as for letrozole monotherapy. ${ }^{6,7}$ A Weibull model was used for both PFS and OS. This generated 10-year PFS and OS rates of $1 \%$ and $15 \%$, respectively.

For palbociclib plus letrozole, survival was modeled using overall response, PFS, and OS for letrozole (as previously detailed), adjusted for the relative efficacy of palbociclib plus letrozole versus letrozole from a meta-analysis of the PALOMA-1 and PALOMA-2 (overall response and PFS only) studies (data on file, Analysis Group, Systematic literature review and network meta-analysis of clinical trials in the firstline setting for advanced HR+/HER2- breast cancer, 2017).
Overall response to therapy was assumed to accrue linearly during the first 12 months of treatment (in line with the results of MONALEESA-2) and to decline with PFS thereafter. ${ }^{6}$

In the base case, the HR of PFS and the odds ratio of response were used. In the absence of patient-level data, OS with palbociclib plus letrozole was assumed to be equal to that of ribociclib plus letrozole. The overall effect of adding a CDK 4/6 to letrozole was to reduce the rates of PFS and OS and increase the rate of response compared with letrozole monotherapy.

Treatment duration for letrozole was modeled on the time spent in the PF health state. Treatment duration with a CDK 4/6 inhibitor was modeled independently of PFS, using data on the time from randomization to treatment discontinuation from MONALEESA-2. ${ }^{6}$ Since comparable discontinuation data were not available from the palbociclib studies, discontinuation for palbociclib was modeled based on the ribociclib data adjusted for any differences in PFS between CDK 4/6 inhibitors ( $\mathrm{HR}=1.010 ; 95 \% \mathrm{CI}=0.730-1.390)$. The comparative efficacy of CDK 4/6 inhibitors was obtained from a Bayesian network meta-analysis (data on file, Analysis Group, Systematic literature review and network meta-analysis of clinical trials in the first-line setting for advanced HR+/HER2- breast cancer, 2017).

\section{Cost Data}

All costs were in 2016 U.S. dollars inflated using the Consumer Price Index. ${ }^{17}$ Only costs relating to direct reimbursable medical care were considered; these included drug acquisition, administration and monitoring, disease management (healthstate costs), subsequent treatments, and adverse events (AEs).

Drug Acquisition Costs. Drug acquisition costs (Table 1) were sourced from Medi-Span Price Rx using the wholesale acquisition cost (WAC) of each medication and applied for the duration that patients remained on therapy in the model..$^{18}$ Annual drug acquisition expenditure was calculated from the WAC and the mean total drug dose administered per year of the simulation (dosing was modeled according to U.S. Food and Drug Administration [FDA] label specifications) minus monthly copayments ( $\$ 567$ for ribociclib plus letrozole or palbociclib plus letrozole [assumed to be equal]; $\$ 11$ for letrozole). The treatment acquisition cost for ribociclib was provided by Novartis.

Drug Administration and Monitoring Costs. Monthly treatment administration costs were taken from the 2016 Physician's Fee Schedule, using Medicare facility prices. ${ }^{19}$ Administration costs were not applied to oral formulations, as these do not require supervised administration.

Drug-specific monitoring costs were applied to ribociclib and palbociclib at treatment initiation and at fixed intervals up to a maximum of 6 or 12 months, respectively, as directed by the product labels. ${ }^{12,14}$ Monitoring requirements for letrozole were captured through routine disease-monitoring costs assigned to the PF and PD health states. 


\section{Cost-Effectiveness of Ribociclib plus Letrozole Versus Palbociclib plus Letrozole and Letrozole Monotherapy in the First-Line Treatment of Postmenopausal Women with HR+/HER2- Advanced or Metastatic Breast Cancer: A U.S. Payer Perspective}

\section{TABLE 1 Summary of Model Inputs}

Input

Let Monotherapy

Pal + Let

Reference

Survival

PFS, HR, mean $(95 \% \mathrm{CI})^{\mathrm{a}}$

OS, HR, mean $(95 \% \mathrm{CI})^{\mathrm{a}}$

OvR, OR, mean $(95 \% \mathrm{CI})^{\mathrm{a}}$

HRs

PFS

Drug acquisition costs per month, \$

CDK 4/6 inhibitor

\section{Letrozole}

Disease management costs per month, $\$$

Outpatient visi

Bone metastases management

Hospitalization

Monitoring (LFT, CBC, and CMP)

Laboratory scan and tests

Bone scan

CT scan

Palliative care

Add-on treatment costs, $\$$

Monitoring at treatment initiation

Monthly monitoring

End-of-life costs, \$

AEs, probability, \%

\begin{tabular}{l}
\hline Diarrhea \\
\hline Fatigue \\
\hline Infection \\
\hline Nausea \\
\hline Febrile neutropenia \\
\hline Pulmonary embolism \\
\hline Vomiting \\
\hline Anemia
\end{tabular}

AEs, unit cost, \$

\begin{tabular}{l|r}
\hline Diarrhea & 7,377
\end{tabular}

Fatigue

Infection

Nausea

Febrile neutropenia

Pulmonary embolism

Vomiting

Anemia

Health-state utility values, mean (SE)

PFS

CR/PR

Stable disease

$\mathrm{PD}$

\begin{tabular}{|c|c|c|c|}
\hline- & \multirow[t]{3}{*}{-} & $0.560 \quad(0.460-0.680)$ & \multirow[t]{3}{*}{ Data on file } \\
\hline $0.840 \quad(0.492-1.345)$ & & $0.840 \quad(0.492-1.345)$ & \\
\hline $1.807(1.320-2.520)$ & & $1.400(1.040-1.890)$ & \\
\hline $1.010 \quad(0.730-1.390)^{\mathrm{c}}$ & - & - & Data on file \\
\hline $600 \mathrm{mg}: 10,950$ & \multirow[t]{3}{*}{-} & \multirow[t]{3}{*}{10,963} & \multirow{3}{*}{$\begin{array}{l}\text { 18, data on } \\
\text { file }\end{array}$} \\
\hline $400 \mathrm{mg}: \quad 8,760$ & & & \\
\hline $200 \mathrm{mg}: 4,380^{\mathrm{d}}$ & & & \\
\hline $8^{f}$ & $8^{f}$ & $8^{f}$ & 18 \\
\hline \multicolumn{2}{|l|}{$\mathrm{PF}$} & PD & \\
\hline \multicolumn{2}{|l|}{47} & 214 & \multirow[t]{8}{*}{17,20} \\
\hline \multicolumn{2}{|l|}{160} & 342 & \\
\hline \multicolumn{2}{|l|}{272} & 703 & \\
\hline \multicolumn{2}{|l|}{15} & - & \\
\hline \multicolumn{2}{|l|}{-} & 420 & \\
\hline \multicolumn{2}{|l|}{54} & - & \\
\hline \multicolumn{2}{|l|}{139} & - & \\
\hline \multirow{2}{*}{ Rib+Let } & & $\begin{array}{c}- \\
4,521 \\
\end{array}$ & \\
\hline & Let Monotherapy & Pal+ Let & \\
\hline 138 & - & 42 & $\begin{array}{c}\text { 37, } 38 \text {, data } \\
\text { on fileg }\end{array}$ \\
\hline \multirow[t]{2}{*}{22} & - & 11 & \\
\hline & 2,392 & & $\begin{array}{c}20, \text { data } \\
\text { on fileg }\end{array}$ \\
\hline 1.2 & 0.9 & 1.7 & \multirow[t]{8}{*}{$6-8$} \\
\hline 2.4 & 0.9 & 2.3 & \\
\hline 4.2 & 2.4 & 4.4 & \\
\hline 2.4 & 0.6 & 0.6 & \\
\hline 1.2 & 0.0 & 0.4 & \\
\hline 0.0 & 0.3 & 5.0 & \\
\hline 3.6 & 0.9 & 0.4 & \\
\hline 1.2 & 1.2 & 5.5 & \\
\hline
\end{tabular}

$\begin{array}{r}7,377 \\ 6,908 \\ \hline 10,128 \\ 6,182 \\ \hline 21,156 \\ \hline 10,036 \\ \hline 5,246 \\ \hline 6,777\end{array}$

$0.837 \quad(0.007)$
$0.830(0.006)$
$0.443^{h}$




\section{Cost-Effectiveness of Ribociclib plus Letrozole Versus Palbociclib plus Letrozole and Letrozole Monotherapy in the First-Line Treatment of Postmenopausal Women with HR+/HER2- Advanced or Metastatic Breast Cancer: A U.S. Payer Perspective}

\section{TABLE 1 Summary of Model Inputs (continued)}

\begin{tabular}{|c|c|c|c|c|}
\hline & Rib + Let & Let Monotherapy & Pal + Let & Reference \\
\hline \multicolumn{5}{|l|}{ AE disutility valuesi } \\
\hline Diarrhea & & -0.060 & & \multirow[t]{8}{*}{6} \\
\hline Fatigue & & -0.029 & & \\
\hline Infection & & -0.050 & & \\
\hline Nausea & & -0.021 & & \\
\hline Febrile neutropenia & & -0.012 & & \\
\hline Pulmonary embolism & & -0.050 & & \\
\hline Vomiting & & -0.050 & & \\
\hline Anemia & & -0.029 & & \\
\hline Discounting rate, $\%$ & & 3.000 & & 28 \\
\hline
\end{tabular}

aVersus letrozole monotherapy.

${ }^{b}$ Data on file, Analysis Group, Systematic literature review and network meta-analysis of clinical trials in the first-line setting for advanced HR+/HER2- breast cancer, 2017.

cVersus palbociclib plus letrozole; used as a surrogate outcome to generate time-to-treatment discontinuation for palbociclib plus letrozole.

dThe model estimates the number of patients on each dose over time using data from MONALEESA-2. This is modeled using the distribution of doses received and the median time to dose reduction during MONALEESA-2. All patients initiate therapy at the $600 \mathrm{mg}$ dose. Between month 0 and the median time to reduction (5.0 months in the base case), the proportion of patients at the $400 \mathrm{mg}$ or $200 \mathrm{mg}$ doses increases at a linear rate. After the median time to reduction, the dose distribution is held constant until the end of the time horizon.

'Data on file, Novartis, e-mail communications, 2016

fWAC price of $\$ 8$; however, with a copayment of $\$ 11$, the acquisition cost is reduced to $\$ 0$.

gData on file, Novartis, Clinical study report: a randomized double-blind, placebo controlled study of LEE011 in combination with letrozole for the treatment of postmenopausal women with hormone receptor positive, HER2-negative, advanced breast cancer who received no prior therapy for advanced disease, 2016.

hStandard error was not reported by Lloyd et al. (2006);26 the model assumes that the standard error is equivalent to $10 \%$ of the mean utility.

${ }^{i}$ A duration of 30 days was applied in each case.

$A E=$ adverse event $C B C=$ complete blood count; $C D K$ 4/6=cyclin-dependent kinase 4 and $6 ; C I=$ confidence interval; $C M P=$ comprehensive metabolic panel;

$C R=$ complete response; $C T=$ computerized tomography; $H R=$ hazard ratio; Let =letrozole; $L F T=$ liver function test; $O R=$ odds ratio; OS =overall survival; OvR=overall response; $\mathrm{Pal}=$ palbociclib; $\mathrm{PD}=$ progressed disease; $\mathrm{PF}=$ progression free; $\mathrm{PFS}=$ progression-free survival; $P R=$ partial response; Rib=ribociclib; $S E=$ standard error.

Health State and Subsequent Treatment Costs. Routine disease management costs, including hospitalizations, outpatient visits, and laboratory scans, were modeled using data from Xie et al. (2015) and applied monthly to the PF and PD states (Table 1). ${ }^{20}$

Subsequent (second- and third-line) treatment costs were applied one-off at the start of the model evaluation. Not all patients were assumed to receive further treatment, and the case mix of subsequent therapies varied depending on whether a CDK 4/6 inhibitor was received at first line, to reflect the availability and expected use of palbociclib at second line after letrozole monotherapy. The use of a CDK 4/6 inhibitor after progression on a CDK 4/6 inhibitor at first line was not considered.

Total cost of subsequent treatment was calculated by multiplying the mean treatment duration in months by the monthly treatment cost and the market shares in a second-line or thirdline setting. Market share and treatment data were sourced from Novartis, and the lowest WAC formulation on the market was used (data on file, Novartis, e-mail communications, 2016). Eribulin was chosen as a proxy representing all chemotherapies because it was the highest-cost first-line chemotherapy.

Adverse Events. The analysis included serious AEs (Grade $\geq 3$ according to the "Common Terminology Criteria for Adverse Events" ${ }^{\prime 2}$ ) that were reported more frequently with palbociclib ${ }^{7}$ or ribociclib ${ }^{6}$ versus placebo and that, from previous health technology assessment submissions and consultation with modeling experts, were deemed relevant and related to the treatment and likely to result in hospitalization or have a meaningful impact on patient well-being (Table 1). The probability of patients experiencing a Grade $\geq 3 \mathrm{AE}$ was obtained from clinical studies. ${ }^{6,722,23}$ Associated costs were derived from the Healthcare Cost and Utilization Project's National Inpatient Sample and were applied one-off at the beginning of the model. ${ }^{24}$ Table 1 provides an overview of AE probabilities and costs.

\section{Utility Data}

Treatment effectiveness was measured in LYs calculated from survival projections and QALYs (calculated from the time spent in each state combined with health-state utility [HSU] values).

Utility values for PF with stable disease and PF with response were derived from EuroQol 5-dimension 5-level (EQ-5D-5L) data collected in MONALEESA-2, which were converted to HSUs using a published value set. Since a U.S. value set for the EQ-5D-5L was not available, HSUs were calculated using the latest UK value set. ${ }^{25}$

Utility values for PD were obtained from the published literature because, in MONALEESA-2, EQ-5D-5L was collected up to only 1 month after progression, thereby limiting data capture to the immediate health consequences of $\mathrm{PD}$. The study by Lloyd et al. (2006) was used; this study reported HSUs 


\section{Cost-Effectiveness of Ribociclib plus Letrozole Versus Palbociclib plus Letrozole and Letrozole Monotherapy in the First-Line Treatment of Postmenopausal Women with HR+/HER2- Advanced or Metastatic Breast Cancer: A U.S. Payer Perspective}

\section{TABLE 2 Total and Incremental Costs and OALYS}

\begin{tabular}{|c|c|c|c|c|c|}
\hline & \multicolumn{3}{|c|}{ Treatment } & \multicolumn{2}{|c|}{ Incremental } \\
\hline & Rib + Let & Let & Pal+ Let & Rib + Let vs. Let & Rib+ Let vs. Pal+ Let \\
\hline \multicolumn{6}{|l|}{ Drug costs, $\$$} \\
\hline CDK 4/6 inhibitor & 228,605 & 0 & 256,393 & 228,605 & $-27,788$ \\
\hline Letrozole & 0 & 0 & 0 & 0 & 0 \\
\hline Monitoring & 196 & 0 & 116 & 196 & 80 \\
\hline Total drug costs & 228,801 & 0 & 256,509 & 228,801 & $-27,708$ \\
\hline \multicolumn{6}{|l|}{ Health-state costs, $\$$} \\
\hline PFS & 21,790 & 12,834 & 19,931 & 8,956 & 1,859 \\
\hline Responder & 9,672 & 3,830 & 7,104 & 5,842 & 2,567 \\
\hline Stable disease & 12,118 & 9,003 & 12,826 & 3,115 & -708 \\
\hline $\mathrm{PD}$ & 150,772 & 187,606 & 167,575 & $-36,834$ & 16,803 \\
\hline Second-line+ & 27,285 & 84,027 & 27,285 & $-56,742$ & 0 \\
\hline AEs & 1,349 & 573 & 1,735 & 776 & -386 \\
\hline End of life & 2,098 & 2,140 & 2,098 & -42 & 0 \\
\hline Total health-state costs & 203,294 & 287,180 & 218,623 & $-83,886$ & $-15,330$ \\
\hline Total cost, $\$$ & 432,095 & 287,180 & 475,132 & 144,915 & $-43,037$ \\
\hline \multicolumn{6}{|l|}{ LYs } \\
\hline $\mathrm{PF}$ & 2.61 & 1.52 & 2.38 & & \\
\hline $\mathrm{PD}$ & 2.03 & 2.52 & 2.26 & & \\
\hline Total LYs & 4.64 & 4.04 & 4.64 & & \\
\hline \multicolumn{6}{|l|}{ QALYS } \\
\hline $\mathrm{PF}$ & 2.17 & 1.26 & 1.99 & & \\
\hline $\mathrm{PD}$ & 0.90 & 1.12 & 1.00 & & \\
\hline Total QALYs & 3.07 & 2.38 & 2.99 & 0.689 & 0.086 \\
\hline ICER & & & & 210,369 & Dominated by Rib + Let \\
\hline
\end{tabular}

estimated via the standard gamble technique and has been used in previous economic studies. ${ }^{26}$

Disutility values associated with AEs (i.e., the effects of AEs on HSU) were also sourced from the literature. Table 1 shows the HSU values applied to the PF and PD health states and the disutility values associated with AEs presented by AE and mean AE duration. ${ }^{27}$

\section{Analyses}

The outputs of the analysis included total and incremental costs and QALYs gained with ribociclib plus letrozole versus palbociclib plus letrozole and versus letrozole monotherapy. Costs and outcomes were discounted at 3\% annually. ${ }^{28}$

Deterministic Sensitivity Analysis. A one-way deterministic sensitivity analysis (DSA) was performed to explore the impact of varying key parameter values on the incremental costeffectiveness ratio (ICER) of ribociclib plus letrozole versus palbociclib plus letrozole and versus letrozole monotherapy. Each key parameter was varied by its standard error, 95\% CI, or $\pm 10 \%$ of the expected (base case) value. Key parameters included discount rate (0\%-6\% variation), treatment acquisition costs $( \pm 10 \%)$ for ribociclib plus letrozole and the selected comparator, health-state (PFS, PD) costs ( $\pm 10 \%)$, AE incidence rates $( \pm 10 \%)$, and utilities for PF and PD health states $( \pm 10 \%)$.

Probabilistic Sensitivity Analysis. Probabilistic uncertainty was assessed through a Monte Carlo probabilistic sensitivity analysis (PSA; $\mathrm{n}=1,000$ iterations). Key parameters included clinical data (survival distributions for PFS and OS and timeto-treatment distribution); cost data (drug acquisition and monitoring costs, disease management costs, and AE costs); and utility data (utility weights assigned to PF and PD states, and disutility of AEs). Multivariate normal distributions with correlation between shape and scale parameter were applied to clinical data, gamma distributions were applied to costs, and beta and gamma distributions were applied to utilities and disutilities, respectively.

Model Assumptions. The following model assumptions were made: (a) Patients received ribociclib plus letrozole or, in accordance with National Comprehensive Cancer Network guidelines, palbociclib plus letrozole or letrozole monotherapy; (b) all costs were applied to the mid-cycle occupancy to minimize underor overestimation; (c) the cost of CDK $4 / 6$ inhibitor therapy was modeled independently of PFS, based on MONALEESA-2 data showing that, on average, patients discontinue 


\section{Cost-Effectiveness of Ribociclib plus Letrozole Versus Palbociclib plus Letrozole and Letrozole Monotherapy in the First-Line Treatment of Postmenopausal Women with HR+/HER2- Advanced or Metastatic Breast Cancer: A U.S. Payer Perspective}

treatment before disease progression; (d) MONALEESA-2 and PALOMA-1 were considered equivalent, so that any difference in patient population had a minimal impact on outcomes; (e) subsequent treatment costs accrued at the start of the time horizon; (f) ribociclib plus letrozole and palbociclib plus letrozole were assumed to have had a clinical equivalence effect on OS and PFS, since the therapies share the same CDK 4/6 pathway inhibition mechanism; $(\mathrm{g})$ proportional hazards between comparators held true (in the absence of patient-level data from other trials, it was challenging to reestimate the effect of treatment on a nonproportional hazards scale); (h) AEs occurred in the first month of the model, reflecting the likelihood that they would be experienced within the first year of therapy; and (i) HSUs were dependent on response status, as supported by the published literature.

\section{Results}

\section{Base Case}

Deterministic Base Case Results. Treatment with ribociclib plus letrozole had a total cost of $\$ 432,095$ (calculated as the sum of drug costs $[\$ 228,801]$ and health-state costs $[\$ 203,294])$ versus $\$ 475,132$ ( $\$ 256,509$ and $\$ 218,623)$ for palbociclib plus letrozole and $\$ 287,180$ ( $\$ 0$ and $\$ 287,180$ ) for letrozole monotherapy (Table 2). Total LYs for each treatment were 4.64 for ribociclib plus letrozole $(\mathrm{PF}=2.61 ; \mathrm{PD}=2.03)$, 4.64 for palbociclib plus letrozole (2.38 and 2.26), and 4.04 for letrozole monotherapy (1.52 and 2.52). Total QALYs for each treatment were 3.07 for ribociclib plus letrozole $(\mathrm{PF}=2.17$; $\mathrm{PD}=0.90), 2.99$ (1.99 and 1.00) for palbociclib plus letrozole, and 2.38 (1.26 and 1.12) for letrozole monotherapy.

In terms of incremental costs and QALYs, ribociclib plus letrozole treatment was associated with a cost reduction of $\$ 43,037$ and a gain of 0.086 QALYs compared with palbociclib plus letrozole; ribociclib plus letrozole was therefore dominant versus palbociclib plus letrozole (Table 2). Compared with letrozole monotherapy, ribociclib plus letrozole was associated with an incremental cost of $\$ 144,915$ and an incremental QALY gain of 0.689 , giving an ICER of $\$ 210,369$ per QALY gained.

\section{Sensitivity Analyses}

According to the DSA, the key model drivers were the OS HR for palbociclib plus letrozole versus letrozole, the OS HR for ribociclib plus letrozole versus letrozole, the PFS HR of palbociclib plus letrozole versus letrozole, PD health-state costs, utility of response, and cost discount rate (Appendix, available in online article).

According to the PSA, ribociclib plus letrozole remained dominant versus palbociclib plus letrozole, with a mean incremental cost reduction of $\$ 45,511$ and a mean increase of 0.040 QALYs. The probabilities that ribociclib plus letrozole were cost-effective versus palbociclib plus letrozole at thresholds of $\$ 50,000, \$ 100,000$ and $\$ 200,000$ per QALY gained were $72.5 \%, 80.7 \%$, and $81.9 \%$, respectively.
In comparison with letrozole, ribociclib plus letrozole resulted in a mean incremental cost of $\$ 143,834$ and a mean incremental QALY of 0.757 , giving an ICER of $\$ 190,001$ per QALY gained. The probabilities that ribociclib plus letrozole were cost-effective versus letrozole at thresholds of $\$ 50,000$, $\$ 100,000$ and $\$ 200,000$ per QALY gained were $1.6 \%, 6.3 \%$, and $50.5 \%$, respectively. The cost-effectiveness acceptability curves for ribociclib plus letrozole versus palbociclib plus letrozole and letrozole monotherapy are presented in Figure 2.

\section{Discussion}

Palbociclib was the first CDK 4/6 inhibitor to receive FDA approval for use in breast cancer in combination with letrozole. Despite the clinical benefits reported in earlier studies, 7,8 recent analyses have failed to demonstrate that palbociclib plus letrozole is cost-effective compared with usual care in the firstline treatment of HR+/HER2- advanced breast cancer. ${ }^{9-11}$ By contrast, the results from the present study indicate that ribociclib is cost-effective compared with letrozole at acceptable thresholds of $\$ 198,000$ per QALY (via probabilistic analysis). Since, in the United States, common thresholds for new cancer technology have increased from $\$ 50,000$ to approximately $\$ 200,000$ over the last few decades, ${ }^{29-31}$ a choice needs to be made at an individual plan level on the willingness to pay per increased unit of health with combination CDK 4/6 and letrozole.

The results of this cost-effectiveness analysis clearly support a preferred placement for ribociclib in combination with letrozole. The results may inform formulary decision making in the current market given the increasing importance of weighing cost-effectiveness analysis alongside clinical evidence. ${ }^{32,33}$ Additionally, it will likely provide a baseline for capturing health outcomes and costs data to validate the model assumptions and outputs within individual health plans once realworld evidence on the use of ribociclib becomes available.

It is noteworthy that the CDK 4/6 inhibitor abemaciclib was not considered as a treatment option in this model because it had not received FDA approval at the point of model development. The subsequent approval included the same patient population but a somewhat different use: in combination with fulvestrant for disease progression following endocrine therapy or as monotherapy for disease progression following endocrine therapy and prior chemotherapy in the metastatic setting. ${ }^{34}$

Our findings are broadly consistent with those of other published work on the cost-effectiveness of CDK 4/6 inhibitors in HR+/HER2- breast cancer. The analysis by Bhattacharya et al. (2016) used a Markov model with PF, PD, palliative care, and death states to assess the cost-effectiveness of palbociclib plus letrozole versus anastrozole monotherapy from a U.S. third-party perspective. ${ }^{35}$ The incremental cost-effectiveness ratio was $\$ 510,356$ per LY gained, leading to the conclusion that palbociclib was unlikely to be cost-effective in the patient population considered for the analysis. 
Cost-Effectiveness of Ribociclib plus Letrozole Versus Palbociclib plus Letrozole and Letrozole Monotherapy in the First-Line Treatment of Postmenopausal Women with HR+/HER2- Advanced or Metastatic Breast Cancer: A U.S. Payer Perspective

\section{FIGURE 2 Cost-Effectiveness Acceptability Curves for Ribociclib plus Letrozole Versus Palbociclib} plus Letrozole and Letrozole Monotherapy

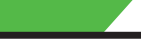

A. Palbociclib plus Letrozole

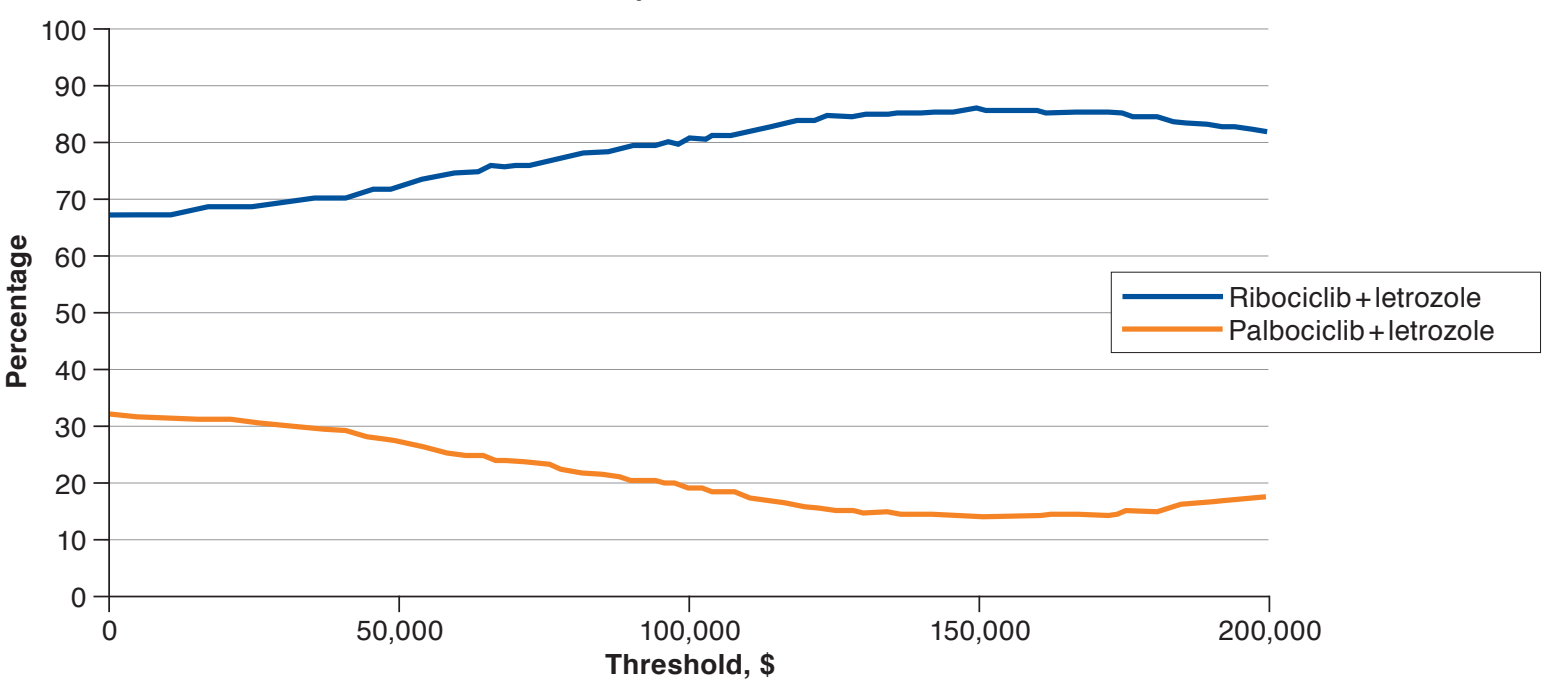

B. Letrozole Monotherapy

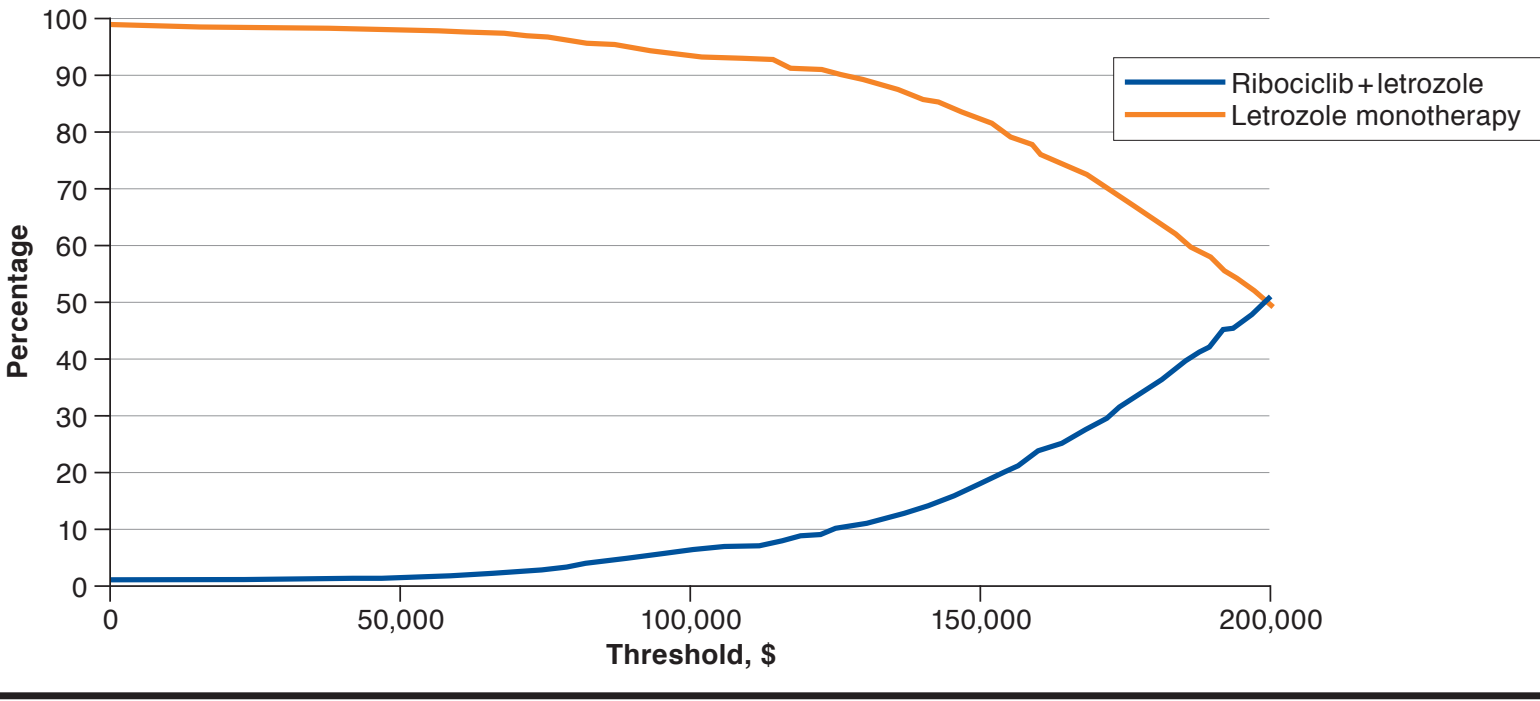

A study by Matter-Walstra et al. (2016) used a 3-state Markov model, similar to the one presented here, to estimate the cost-effectiveness of palbociclib plus letrozole versus letrozole monotherapy from a Swiss health care perspective. ${ }^{10}$ Clinical efficacy was based on PALOMA-1, with PFS and OS extrapolated using exponential distributions. The incremental cost-effectiveness ratio was 301,227 CHF (Swiss francs) per QALY gained, with undiscounted QALYs of 3.33 for palbociclib plus letrozole and 2.19 for letrozole monotherapy.

The authors concluded that palbociclib was unlikely to be cost-effective in Switzerland. Notably, their QALY predictions are consistent with values reported here, further validating our findings, although cost and ICER results cannot be generalized to a U.S. health care setting, due to differences in perspective (i.e., public health care versus third-party payer) and currency (Swiss francs versus U.S. dollars).

Our economic model has a number of strengths. The main data source was MONALEESA-2, a large randomized phase III study that enrolled patients from the United States. ${ }^{6}$ Where required, MONALEESA-2 was supplemented by data from other studies.,22,23 A key driver of the analysis, the cost of CDK 4/6 inhibitor treatment, was also modeled directly from 


\section{Cost-Effectiveness of Ribociclib plus Letrozole Versus Palbociclib plus Letrozole and Letrozole Monotherapy in the First-Line Treatment of Postmenopausal Women with HR+/HER2- Advanced or Metastatic Breast Cancer: A U.S. Payer Perspective}

exposure data in MONALEESA-2, providing an accurate prediction of drug costs. Other models have used PFS as a proxy of treatment discontinuation, which may overestimate cost if discontinuation occurs before progression. EQ-5D-5L data from MONALEESA-2 were also used for HSU and are considered representative of the health-related QoL for the target population for ribociclib plus letrozole.

\section{Limitations}

This study has limitations that are intrinsic to many modeling efforts. Relevant clinical trials reporting PFS and OS in HR+/ HER2- first-line breast cancer are scarce and show statistical and clinical heterogeneity stemming from differences in study design, particularly dose exposure. The mixing of PFS and OS from 2 separate trials (MONALEESA-2 and PALOMA-1, respectively $)^{6,7}$ relies on the assumption that the 2 sources are generalizable to each other, which may not hold true if one population is healthier than another or has access to more efficacious subsequent treatments. The extent and direction of any bias from the mixing of studies is unclear, given that this assumption applied to all strategies in the analysis.

There are also limitations in the availability of applicable health care resource use data, and of HSU from a U.S. societal perspective. In this analysis, it was necessary to source resource use data from an economic evaluation in second-line breast can$\mathrm{cer}^{20}$ and to use HSU generated from UK societal preferences ${ }^{6}$ combined with standard gamble HSU. ${ }^{26}$ The extent to which these data sources adequately represent the costs and QoL of first-line patients in the United States is unclear. Further research on the costs and preferences of U.S. patients with postmenopausal HR+/HER2- breast cancer would help reduce uncertainty surrounding future evaluations in this area.

\section{Conclusions}

As the already substantial economic burden imposed by breast cancer in the United States is projected to increase to $\$ 20.5$ billion by $2020,{ }^{36}$ there is a need for new therapy options that improve patient outcomes in a cost-effective manner.

Based on the results of this economic analysis, firstline treatment of postmenopausal women with HR+/HER2advanced or metastatic breast cancer with ribociclib $\mathrm{p}$

lus letrozole generates QALY gains and cost savings compared with palbociclib plus letrozole therapy. Therefore, the combination ribociclib plus letrozole provides increased money value and represents an efficient use of resources by thirdparty payers in the United States. Furthermore, our economic analysis shows that, at willingness-to-pay thresholds above $\$ 198,000$ per QALY (for probabilistic analysis), treatment with ribociclib plus letrozole is also cost-effective versus letrozole monotherapy.

\section{Authors}

ROHIT MISTRY, MPharm, MBA, MSc; JESSICA R. MAY, PhD; and GAURAV SURI, MSc, BTech, PAREXEL, London, United Kingdom. KATE YOUNG, PhD, MPH, PAREXEL, Horsham, Pennsylvania. DIANA BRIXNER, PhD, RPh, FAMCP; GARY ODERDA, PharmD, MPH; and JOSEPH BISKUPIAK, PhD, MBA, University of Utah, Salt Lake City. DEREK TANG, PhD, BSPharm, and ANAND A. DALAL, PhD, MBA, Novartis, East Hanover, New Jersey. SUBRATA BHATTACHARYYA, MSc; DINESH MISHRA, PhD, MSc; and DEVARSHI BHATTACHARYYA, MPH, MSc, BDS, Novartis, Hyderabad, Telangana, India.

AUTHOR CORRESPONDENCE: Gaurav Suri, MSc, BTech, PAREXEL, Evergreen Building N., 160 Euston Rd., London NW1 2DX, United Kingdom. Tel.: +44 207.121.1762;

E-mail: gaurav.suri@parexel.com.

\section{DISCLOSURES}

Funding for this study was provided by Novartis, which manufactures ribociclib and provided input on the study design and data collection, analysis, and interpretation. Mistry, May, Suri, and Young are employees of PAREXEL. Tang, Mishra, D. Bhattacharyya, and Dalal are employees of Novartis. S. Bhattacharyya was an employee of Novartis during the study period. Tang and Dalal hold stock in Novartis. Brixner, Oderda, and Biskupiak were paid by Millcreek Outcomes Group as consultants for work on this project. Brixner has also consulted for AstraZeneca, UCB, Regeneron, and Abbott.

\section{ACKNOWLEDGMENTS}

Editorial support was provided by Lorena Tonarelli, MSc, and Nicholas Rusbridge, PhD, of PAREXEL, London, United Kingdom.

\section{REFERENCES}

1. Howlader N, Altekruse SF, Li CI, et al. U.S. incidence of breast cancer subtypes defined by joint hormone receptor and HER2 status. J Natl Cancer Inst. 2014;106(5).

2. Cardoso F, Costa A, Senkus E, et al. 3rd ESO-ESMO International Consensus Guidelines for Advanced Breast Cancer (ABC 3). Ann Oncol. 2017;28(1):16-33.

3. Gradishar WJ, Anderson BO, Balassanian R, et al. Invasive breast cancer version 1.2016, NCCN clinical practice guidelines in oncology. J Natl Compr Canc Netw. 2016;14(3):324-54.

4. Cardoso F, Costa A, Norton L, et al. ESO-ESMO 2nd international consensus guidelines for advanced breast cancer (ABC2). Breast. 2014;23(5):489-502.

5. Tripathy D, Bardia A, Sellers WR. Ribociclib (LEE011): mechanism of action and clinical impact of this selective cyclin-dependent kinase 4/6 inhibitor in various solid tumors. Clin Cancer Res. 2017;23(13):3251-62.

6. Hortobagyi GN, Stemmer SM, Burris HA, et al. Ribociclib as firstline therapy for HR-positive, advanced breast cancer. N Engl J Med. 2016;375(18):1738-48.

7. Finn RS, Crown JP, Lang I, et al. The cyclin-dependent kinase 4/6 inhibitor palbociclib in combination with letrozole versus letrozole alone as firstline treatment of oestrogen receptor-positive, HER2-negative, advanced breast cancer (PALOMA-1/TRIO-18): a randomised phase 2 study. Lancet Oncol. 2015;16(1):25-35. 


\section{Cost-Effectiveness of Ribociclib plus Letrozole Versus Palbociclib plus Letrozole and Letrozole Monotherapy in the First-Line Treatment of Postmenopausal Women with HR+/HER2- Advanced or Metastatic Breast Cancer: A U.S. Payer Perspective}

8. Finn RS, Martin M, Rugo HS, et al. PALOMA-2: primary results from a phase III trial of palbociclib (P) with letrozole (L) compared with letrozole alone in postmenopausal women with ER+/HER2- advanced breast cancer (ABC). J Clin Oncol. 2016;34(15 Suppl):507. Available at: http://ascopubs.org/ doi/abs/10.1200/JCO.2016.34.15_suppl.507. Accessed May 15, 2018.

9. Mamiya H, Tahara RK, Tolaney SM, Choudhry N, Najafzadeh M. Costeffectiveness of palbociclib in hormone receptor-positive advanced breast cancer. Ann Oncol. 2017;28(8):1825-31.

10. Matter-Walstra K, Ruhstaller T, Klingbiel D, Schwenkglenks M, Dedes KJ. Palbociclib as a first-line treatment in oestrogen receptor-positive, HER2negative, advanced breast cancer not cost-effective with current pricing: a health economic analysis of the Swiss Group for Clinical Cancer Research (SAKK). Breast Cancer Res Treat. 2016;158(1):51-57.

11. Matter-Walstra K, Schwenkglenks M, Dedes KJ. Cost-effectiveness of palbociclib plus letrozole versus letrozole alone as a first-line treatment in women with oestrogen receptor-positive, HER2-negative, advanced breast cancer. Revised results for the Swiss health care setting. Breast Cancer Res Treat. 2017;163(3):635.

12. Kisqali (ribociclib) for oral use. Novartis. March 2017. Available at: https://www.accessdata.fda.gov/drugsatfda_docs/label/2017/209092s000lbl. pdf. Accessed April 21, 2018.

13. Glasziou PP, Simes RJ, Gelber RD. Quality adjusted survival analysis. Stat Med. 1990;9(11):1259-76.

14. Ibrance (palbociclib) for oral use. Pfizer. Revised March 31, 2017. Available at: https://www.accessdata.fda.gov/drugsatfda_docs/ label/2017/207103s004lbl.pdf. Accessed April 21, 2018.

15. Tevaarwerk AJ, Gray RJ, Schneider BP, et al. Survival in patients with metastatic recurrent breast cancer after adjuvant chemotherapy: little evidence of improvement over the past 30 years. Cancer. 2013;119(6):1140-48.

16. Weide R, Feiten S, Friesenhahn V, et al. Metastatic breast cancer: prolongation of survival in routine care is restricted to hormone-receptor- and Her2-positive tumors. Springerplus. 2014;3:535.

17. U.S. Bureau of Labor Statistics. Consumer Price Index for all urban consumers: medical care. 2016. Available at: https://data.bls.gov/timeseries/ CUUR0000SAM?output_view=pct_12mths. Accessed April 22, 2018.

18. Medi-Span Price Rx. Wolters Kluwer. Indianapolis, IN. 2016. Available at: https://pricerx.medispan.com/IngredientName.aspx. Accessed April 22, 2018.

19. Centers for Medicare \& Medicaid Services. Physician's fee schedule. 2016. Available at: https://www.cms.gov/apps/physician-fee-schedule/ license-agreement.aspx. Accessed May 15, 2018.

20. Xie J, Hao Y, Zhou ZY, Qi CZ, De G, Gluck S. Economic evaluations of everolimus versus other hormonal therapies in the treatment of HR+/HER2advanced breast cancer from a U.S. payer perspective. Clin Breast Cancer. 2015;15(5):e263-76.

21. National Institutes of Health, National Cancer Institute. Common Terminology Criteria for Adverse Events v.3.0 (CTCAE). August 9, 2006. Available at: https://ctep.cancer.gov/protocoldevelopment/electronic_applications/docs/ctcaev3.pdf. Accessed May 15, 2018.

22. Johnston SR, Kilburn LS, Ellis P, et al. Fulvestrant plus anastrozole or placebo versus exemestane alone after progression on non-steroidal aromatase inhibitors in postmenopausal patients with hormone-receptor-positive locally advanced or metastatic breast cancer (SoFEA): a composite, multicentre, phase 3 randomised trial. Lancet Oncol. 2013;14(10):989-98.
23. Turner NC, Ro J, Andre F, et al. Palbociclib in hormone-receptor-positive advanced breast cancer. N Engl J Med. 2015;373(3):209-19.

24. Agency for Healthcare Research and Quality. Healthcare Cost and Utilization Project. National Inpatient Sample, 2013. Available at: https:// hcup-us.ahrq.gov/db/nation/nis/NIS_Introduction_2013.jsp. Accessed April 21, 2018.

25. Devlin NJ, Shah KK, Feng Y, Mulhern B, van Hout B. Valuing healthrelated quality of life: an EQ-5D-5L value set for England. Health Econ. 2018;27(1):7-22.

26. Lloyd A, Nafees B, Narewska J, Dewilde S, Watkins J. Health state utilities for metastatic breast cancer. Br J Cancer. 2006;95(6):683-90.

27. Hudgens S, Briggs A, Tremblay G, Forsythe A, Lloyd A. Comparison of methods to estimate health state utilities in metastatic breast cancer (MBC) Value Health. 2014;17(7):A557.

28. Gold M. Panel on cost-effectiveness in health and medicine. Med Care. 1996;34(12 Suppl):DS197-99.

29. Neumann PJ, Cohen JT, Weinstein MC. Updating cost-effectiveness-the curious resilience of the \$50,000-per-QALY threshold. N Engl J Med. 2014;371(9):796-97.

30. Dilla T, Lizan L, Paz S, et al. Do new cancer drugs offer good value for money? The perspectives of oncologists, health care policy makers, patients, and the general population. Patient Prefer Adherence. 2016;10:1-7.

31. Bae YHJ, Mullins CD. Do value thresholds for oncology drugs differ from nononcology drugs? J Manag Care Spec Pharm. 2014;20(11):1086-92. Available at: https://www.jmcp.org/doi/10.18553/jmcp.2014.20.11.1086.

32. Anbil P, Bajpai S. Formulary decisions without comparative effectiveness research. Manag Care. 2014;23(10):38-41.

33. Wang Z, Salmon JW, Walton SM. Cost-effectiveness analysis and the formulary decision-making process. J Manag Care Pharm. 2004;10(1):48-59. Available at: https://www.jmcp.org/doi/abs/10.18553/jmcp.2004.10.1.48.

34. Verzenio (abemaciclib) for oral use. Eli Lilly. September 2017. Available at: https://www.accessdata.fda.gov/drugsatfda_docs/ label/2017/208716s000lbl.pdf. Accessed on April 21, 2018.

35. Bhattacharya K, Yang Y. A cost-effectiveness analysis of palbociclib and other aromatase inhibitors for treatment of advanced breast cancer. Value Health. 2016;19(3):A150.

36. Mariotto AB, Yabroff KR, Shao Y, Feuer EJ, Brown ML. Projections of the cost of cancer care in the United States: 2010-2020. J Natl Cancer Inst. 2011;103(2):117-28

37. Healthcare Bluebook. 2016. Available at: https://www.healthcarebluebook.com/. Accessed April 21, 2018.

38. European Medicines Agency. Ibrance (palbociclib). 2016. Available at: http://www.ema.europa.eu/docs/en_GB/document_library/EPAR_Summary_for_the_public/human/003853/WC500217199.pdf. Accessed April 21, 2018.

39. Michels SL, Barron RL, Reynolds MW, Smoyer Tomic K, Yu J, Lyman GH. Costs associated with febrile neutropenia in the U.S. Pharmacoeconomics. 2012;30(9):809-23. 
Cost-Effectiveness of Ribociclib plus Letrozole Versus Palbociclib plus Letrozole and Letrozole Monotherapy in the First-Line Treatment of Postmenopausal Women with HR+/HER2- Advanced or Metastatic Breast Cancer: A U.S. Payer Perspective

\section{APPENDIX Key Model Drivers from the Deterministic Sensitivity Analysis of Incremental Cost-Effectiveness} Ratio and Net Monetary Benefit
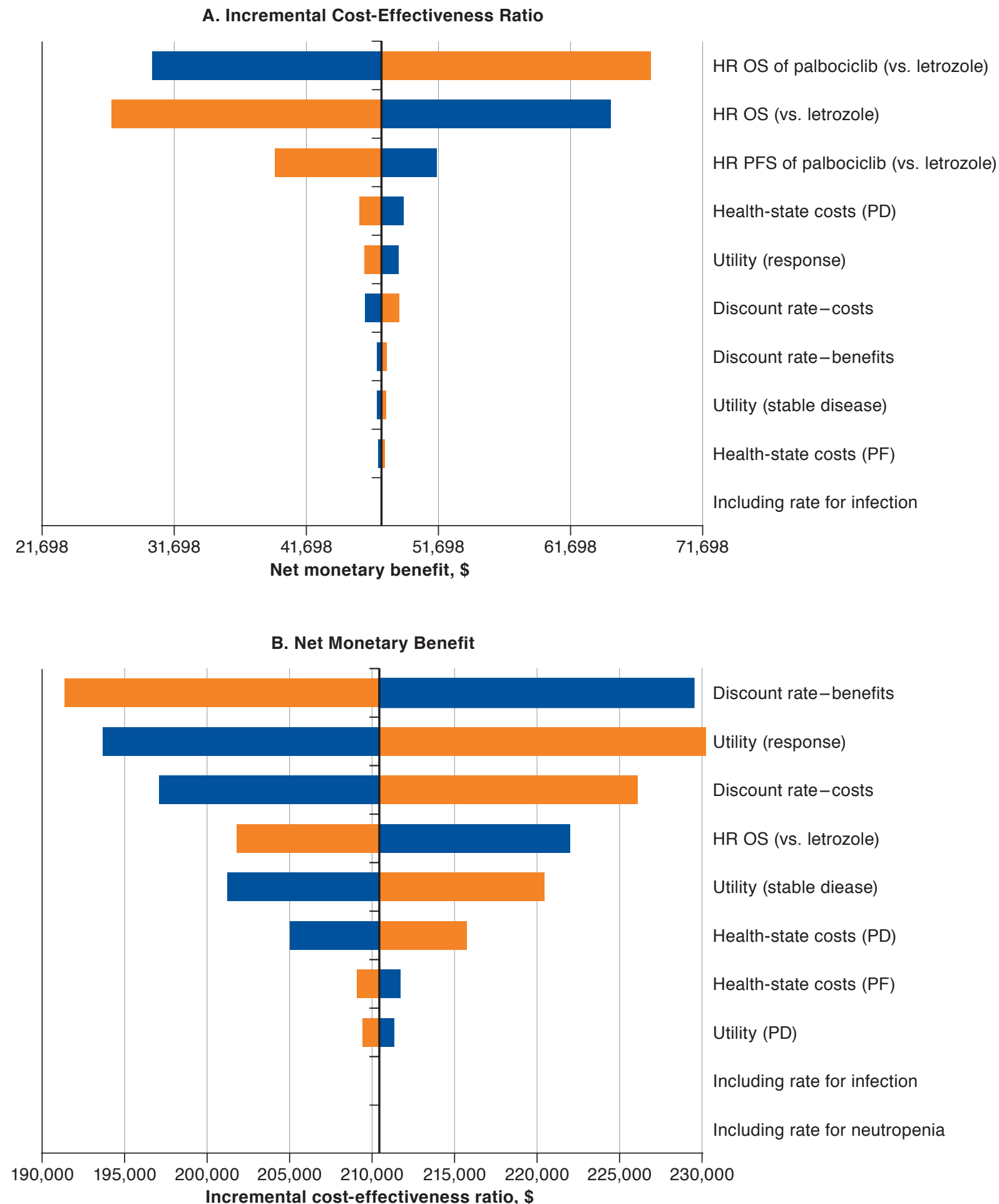

HR = hazard ratio; $O S=$ overall survival; $P D=$ progressed disease PF = progression free PFS = progression-free survival. 